International Journal of Pure and Applied Mathematics

Volume 84 No. 4 2013, 423-434

ISSN: 1311-8080 (printed version); ISSN: 1314-3395 (on-line version)

url: http://www.ijpam.eu

doi: http://dx.doi.org/10.12732/ijpam.v84i4.12

ijpam.eu

\title{
ON APPLICATIONS OF DIFFERENTIAL SUBORDINATION
}

\author{
A. Selvam ${ }^{1}$, P. Sooriya Kala ${ }^{2}$, N. Marikkannan ${ }^{3} \S$ \\ 1,2 Department of Mathematics \\ VHNSN College \\ Virudhunagar, 626001, INDIA \\ ${ }^{3}$ Department of Mathematics \\ Government Arts College \\ Melur, 625106, INDIA
}

\begin{abstract}
Using a generalized differential operator we define certain subclasses of analytic functions and study about their inclusion relationships using differential subordination.
\end{abstract}

AMS Subject Classification: $30 \mathrm{C} 45,30 \mathrm{C} 80$

Key Words: analytic functions, Hadamard products, starlike functions, convex functions, differential subordination and linear operators

\section{Introduction}

Let $\mathcal{A}$ denote the class of functions of the form

$$
f(z):=z+\sum_{k=2}^{\infty} a_{k} z^{k}, \quad a_{k} \geq 0
$$

which are analytic in the open unit disk $U=\{z \in \mathbb{C}:|z|<1\}$. Let $S, S^{*}(\alpha)$ and $K(\alpha)$ denote the subclasses of $\mathcal{A}$ consisting of functions that are univalent, starlike of order $\alpha$ and convexlike of order $\alpha$ respectively. Also $S^{*}(0)=S^{*}$ and $K(0)=K$ are the class of starlike and convex functions defined on $U$ respectively. For two functions $f(z)$ given by (1.1) and $g(z)=z+\sum_{k=2}^{\infty} b_{k} z^{k}$,

Received: January 16, 2013

(C) 2013 Academic Publications, Ltd. url: www.acadpubl.eu

${ }^{\S}$ Correspondence author 
the Hadamard product or convolution of $f$ and $g$ is denoted by $(f * g)(z)$, defined as

$$
(f * g)(z):=z+\sum_{k=2}^{\infty} a_{k} b_{k} z^{k} .
$$

For complex numbers $\alpha_{1}, \alpha_{2}, \ldots, \alpha_{q}$ and $\beta_{1}, \beta_{2}, \ldots, \beta_{s} ;\left(\beta_{j} \in \mathbb{C} \backslash \mathcal{Z}_{0}^{-} ; \mathcal{Z}_{0}^{-}=\right.$ $\{0,-1,-2, \ldots\}$ for $j=1,2, . ., s)$, we define the generalized hypergeometric function as

$$
\begin{gathered}
{ }_{q} F_{s}\left(\alpha_{1}, \alpha_{2}, \ldots, \alpha_{q} ; \beta_{1}, \beta_{2}, \ldots, \beta_{s} ; z\right):=\sum_{k=0}^{\infty} \frac{\left(\alpha_{1}\right)_{k}\left(\alpha_{2}\right)_{k} \ldots\left(\alpha_{q}\right)_{k} z^{k}}{\left(\beta_{1}\right)_{k}\left(\beta_{2}\right)_{k} \ldots\left(\beta_{s}\right)_{k} k !} \\
\left(q \leq s+1 ; q, s \in \mathbb{N}_{0}:=\mathbb{N} \cup\{0\} ; z \in U\right)
\end{gathered}
$$

where $\mathbb{N}$ denotes the set of all positive integers and $(x)_{k}$ is the Pochhammer symbol defined in terms of gamma function, as

$$
(x)_{k}=\frac{\Gamma(x+k)}{\Gamma(x)}=\left\{\begin{array}{lll}
1 & \text { if } & k=0 \\
x(x+1) \ldots(x+k-1) & \text { if } \quad k \in \mathbb{N}
\end{array}\right.
$$

Corresponding to the function $g_{q, s}\left(\alpha_{1}, \beta_{1} ; z\right)$, defined by

$$
g_{q, s}\left(\alpha_{1}, \beta_{1} ; z\right):=z_{q} F_{s}\left(\alpha_{1}, \alpha_{2}, \ldots, \alpha_{q} ; \beta_{1}, \beta_{2}, \ldots, \beta_{s} ; z\right),
$$

recently in [9] an operator $\mathcal{D}_{\lambda, \mu}^{m}\left(\alpha_{1}, \beta_{1}\right) f(z): \mathcal{A} \rightarrow \mathcal{A}$ has been defined by

$$
\begin{aligned}
\mathcal{D}_{\lambda, \mu}^{0}\left(\alpha_{1}, \beta_{1}\right) f(z):= & f(z) * g_{q, s}\left(\alpha_{1}, \beta_{1} ; z\right) \\
\mathcal{D}_{\lambda, \mu}^{1}\left(\alpha_{1}, \beta_{1}\right) f(z):= & (1-\lambda+\mu)\left(f(z) * g_{q, s}\left(\alpha_{1}, \beta_{1} ; z\right)\right) \\
& +(\lambda-\mu) z\left(f(z) * g_{q, s}\left(\alpha_{1}, \beta_{1} ; z\right)\right)^{\prime} \\
& +\lambda \mu z^{2}\left(f(z) * g_{q, s}\left(\alpha_{1}, \beta_{1} ; z\right)^{\prime \prime}\right. \\
\mathcal{D}_{\lambda, \mu}^{m}\left(\alpha_{1}, \beta_{1}\right) f(z):= & \mathcal{D}_{\lambda, \mu}^{1}\left(\mathcal{D}_{\lambda, \mu}^{m-1}\left(\alpha_{1}, \beta_{1}\right) f(z)\right),
\end{aligned}
$$

where $0 \leq \mu \leq \lambda \leq 1$ and $m \in \mathbb{N}_{0}$. By using the above definition, we can find that

$$
\begin{aligned}
& \mathcal{D}_{\lambda, \mu}^{m}\left(\alpha_{1}, \beta_{1}\right) f(z)=z \\
& \quad+\sum_{k=2}^{\infty}[1+(k-1)(\lambda-\mu+k \mu \lambda)]^{m} \frac{\left(\alpha_{1}\right)_{k-1}\left(\alpha_{2}\right)_{k-1} \ldots\left(\alpha_{q}\right)_{k-1}}{\left(\beta_{1}\right)_{k-1}\left(\beta_{2}\right)_{k-1} \ldots\left(\beta_{s}\right)_{k-1}(k-1) !} a_{k} z^{k} .
\end{aligned}
$$

For brevity, let us take

$$
B_{k}=\frac{\left(\alpha_{1}\right)_{k-1}\left(\alpha_{2}\right)_{k-1} \ldots\left(\alpha_{q}\right)_{k-1}}{\left(\beta_{1}\right)_{k-1}\left(\beta_{2}\right)_{k-1} \ldots\left(\beta_{s}\right)_{k-1}(k-1) !} .
$$


Hence we have

$$
\mathcal{D}_{\lambda, \mu}^{m}\left(\alpha_{1}, \beta_{1}\right) f(z)=z+\sum_{k=2}^{\infty}[1+(k-1)(\lambda-\mu+k \mu \lambda)]^{m} B_{k} a_{k} z^{k} .
$$

For suitable values of $\alpha_{i}^{\prime} s, \beta_{j}^{\prime} s, q, s, \lambda$ and $\mu$ we can deduce several operators $[1,6,14]$ as a special case of this operator. Also a simple computation shows that

$$
\begin{gathered}
(1-\gamma) \mathcal{D}_{\lambda, \mu}^{m}\left(\alpha_{1}, \beta_{1}\right) f(z)+\gamma z\left[\mathcal{D}_{\lambda, \mu}^{m}\left(\alpha_{1}, \beta_{1}\right) f(z)\right]^{\prime}=\gamma \alpha_{1} \mathcal{D}_{\lambda, \mu}^{m}\left(\alpha_{1}+1, \beta_{1}\right) f(z) \\
-\left(\gamma \alpha_{1}-1\right) \mathcal{D}_{\lambda, \mu}^{m}\left(\alpha_{1}, \beta_{1}\right) f(z)
\end{gathered}
$$

Let $f(z)$ and $g(z)$ be analytic in the unit disc $U$. Then $f(z)$ is said to be subordinate to $g(z)$ in $U$, if there exists a Schwarz function $w(z)$, analytic in $U$ with $w(0)=0$ and $|w(z)|<1$ for $z \in U$, such that $f(z)=g(w(z))$. We denote it as $f \prec g$. Further if $g(z)$ is univalent then we write $f \prec g$ if $f(0)=g(0)$ and $f(U) \subset g(U)$.

Definition 1.1. Let $h(z)$ be an analytic convex univalent function in $U$ with $h(0)=1$ and $\Re\{h(z)\}>0$ for $z \in U$. Let $A\left(\alpha_{1}, \beta_{1}, \gamma, \lambda, \mu, m, h\right)$ denote the subclass of $\mathcal{A}$ consisting of functions $f(z)$ which satisfy the condition

$$
\frac{z\left[\mathcal{D}_{\lambda, \mu}^{m}\left(\alpha_{1}, \beta_{1}\right) f(z)\right]^{\prime}+\gamma z^{2}\left[\mathcal{D}_{\lambda, \mu}^{m}\left(\alpha_{1}, \beta_{1}\right) f(z)\right]^{\prime \prime}}{(1-\gamma) \mathcal{D}_{\lambda, \mu}^{m}\left(\alpha_{1}, \beta_{1}\right) f(z)+\gamma z\left[\mathcal{D}_{\lambda, \mu}^{m}\left(\alpha_{1}, \beta_{1}\right) f(z)\right]^{\prime}} \prec h(z)
$$

for some $\gamma(0 \leq \gamma \leq 1)$ and for all $z \in U$.

Definition 1.2. Let $h(z)$ be an analytic convex univalent function in $U$ with $h(0)=1$ and $\Re\{h(z)\}>0$ for $z \in U$. Let $B\left(\alpha_{1}, \beta_{1}, \gamma, \lambda, \mu, m, h\right)$ denote the subclass of $\mathcal{A}$ consisting of functions $f(z)$ which satisfy the condition

$$
(1-\gamma) \frac{\mathcal{D}_{\lambda, \mu}^{m}\left(\alpha_{1}, \beta_{1}\right) f(z)}{z}+\gamma\left[\mathcal{D}_{\lambda, \mu}^{m}\left(\alpha_{1}, \beta_{1}\right) f(z)\right]^{\prime} \prec h(z)
$$

for some $\gamma(0 \leq \gamma \leq 1)$ and for all $z \in U$.

Definition 1.3. Let $h(z)$ be an analytic convex univalent function in $U$ with $h(0)=1$ and $\Re\{h(z)\}>0$ for $z \in U$. Let $C\left(\alpha_{1}, \beta_{1}, \gamma, \lambda, \mu, m, h\right)$ denote the subclass of $\mathcal{A}$ consisting of functions $f(z)$ which satisfy the condition

$$
\left[\mathcal{D}_{\lambda, \mu}^{m}\left(\alpha_{1}, \beta_{1}\right) f(z)\right]^{\prime}+\gamma z\left[\mathcal{D}_{\lambda, \mu}^{m}\left(\alpha_{1}, \beta_{1}\right) f(z)\right]^{\prime \prime} \prec h(z)
$$

for some $\gamma(0 \leq \gamma \leq 1)$ and for all $z \in U$. 
Note that special cases of $A\left(\alpha_{1}, \beta_{1}, \gamma, \lambda, \mu, m, h\right), B\left(\alpha_{1}, \beta_{1}, \gamma, \lambda, \mu, m, h\right)$ and $C\left(\alpha_{1}, \beta_{1}, \gamma, \lambda, \mu, m, h\right)$ include $S^{*}, K, S^{*}(\alpha), K(\alpha)$ and many other subclasses of $\mathcal{A}$ which were defined earlier in $[11,12,13]$. By specializing the parameters we get the corresponding classes containing Hohlov operator, Ruscheweyh operator, fractional calculus operator, Sălăgean derivative operator, LiberaBernardi-Livingston integral operator, Dziok-Srivatsava operator and the operator studied in [15].

\section{Preliminaries}

To prove our main results we need the following lemmas.

Lemma 2.1. [10, p. 81] Let $h$ be analytic, univalent and convex in $U$ with $h(0)=1$ and $\Re\{\beta h(z)+\gamma\}>0,(\beta, \gamma \in \mathbb{C}, z \in U)$. If $p$ is analytic in $U$ with $p(0)=h(0)$, then

$$
p(z)+\frac{z p^{\prime}(z)}{\beta p(z)+\gamma} \prec h(z)
$$

implies that

$$
p(z) \prec h(z) \quad(z \in U) .
$$

Lemma 2.2. [10, p. 71] Let $h$ be analytic, univalent and convex in $U$ with $h(0)=1$. Also let $p$ be analytic in $U$ with $p(0)=h(0)$. If

$$
p(z)+\frac{z p^{\prime}(z)}{\gamma} \prec h(z)
$$

then

$$
p(z) \prec q(z) \prec h(z),
$$

where

$$
q(z)=\frac{\gamma}{z^{\gamma}} \int_{0}^{z} t^{\gamma-1} h(t) d t \quad(z \in U, \Re\{\gamma\} \geq 0 ; \gamma \neq 0\}
$$

\section{Inclusion Relations}

Theorem 3.1. For $\alpha_{1} \geq 1$,

$$
A\left(\alpha_{1}+1, \beta_{1}, \gamma, \lambda, \mu, m, h\right) \subset A\left(\alpha_{1}, \beta_{1}, \gamma, \lambda, \mu, m, h\right) .
$$


Proof. Let $f(z) \in A\left(\alpha_{1}+1, \beta_{1}, \gamma, \lambda, \mu, m, h\right)$ and let

$$
p(z):=\frac{z\left[\mathcal{D}_{\lambda, \mu}^{m}\left(\alpha_{1}, \beta_{1}\right) f(z)\right]^{\prime}+\gamma z^{2}\left[\mathcal{D}_{\lambda, \mu}^{m}\left(\alpha_{1}, \beta_{1}\right) f(z)\right]^{\prime \prime}}{(1-\gamma) \mathcal{D}_{\lambda, \mu}^{m}\left(\alpha_{1}, \beta_{1}\right) f(z)+\gamma z\left[\mathcal{D}_{\lambda, \mu}^{m}\left(\alpha_{1}, \beta_{1}\right) f(z)\right]^{\prime}} .
$$

By differentiating (1.2) we have,

$$
p(z)+(a-1)=\frac{\gamma \alpha_{1} z\left[\mathcal{D}_{\lambda, \mu}^{m}\left(\alpha_{1}+1, \beta_{1}\right) f(z)\right]^{\prime}+(1-\gamma) \alpha_{1} \mathcal{D}_{\lambda, \mu}^{m}\left(\alpha_{1}, \beta_{1}\right) f(z)}{(1-\gamma) \mathcal{D}_{\lambda, \mu}^{m}\left(\alpha_{1}, \beta_{1}\right) f(z)+\gamma z\left[\mathcal{D}_{\lambda, \mu}^{m}\left(\alpha_{1}, \beta_{1}\right) f(z)\right]^{\prime}} .
$$

Taking logarithmic differentiation on both sides we get

$$
\begin{aligned}
p(z)+\frac{z p^{\prime}(z)}{p(z)+\left(\alpha_{1}-1\right)} & \\
& =\frac{z\left[\mathcal{D}_{\lambda, \mu}^{m}\left(\alpha_{1}+1, \beta_{1}\right) f(z)\right]^{\prime}+\gamma z^{2}\left[\mathcal{D}_{\lambda, \mu}^{m}\left(\alpha_{1}+1, \beta_{1}\right) f(z)\right]^{\prime \prime}}{(1-\gamma) \mathcal{D}_{\lambda, \mu}^{m}\left(\alpha_{1}+1, \beta_{1}\right) f(z)+\gamma z\left[\mathcal{D}_{\lambda, \mu}^{m}\left(\alpha_{1}+1, \beta_{1}\right) f(z)\right]^{\prime}} .
\end{aligned}
$$

As $f(z) \in A\left(\alpha_{1}+1, \beta_{1}, \gamma, \lambda, \mu, m, h\right)$ we have

$$
p(z)+\frac{z p^{\prime}(z)}{p(z)+\left(\alpha_{1}-1\right)} \prec h(z) .
$$

It follows from Lemma 2.1 that

$$
p(z) \prec h(z)
$$

for $\alpha_{1} \geq 1$. Thus $f \in A\left(\alpha_{1}, \beta_{1}, \gamma, \lambda, \mu, m, h\right)$.

Theorem 3.2. If $f \in A\left(\alpha_{1}, \beta_{1}, \gamma, \lambda, \mu, m, h\right)$ for $\alpha_{1} \geq 1$, then $F_{c}(f) \in$ $A\left(\alpha_{1}, \beta_{1}, \gamma, \lambda, \mu, m, h\right)$, where $F_{c}$ is the integral operator defined by

$$
F_{c}(f)=F_{c}(f)(z):=\frac{c+1}{z^{c}} \int_{0}^{z} t^{c-1} f(t) d t \quad(c \geq 0) .
$$

Proof. Let $f \in A\left(\alpha_{1}, \beta_{1}, \gamma, \lambda, \mu, m, h\right)$ and

$$
p(z):=\frac{z\left[\mathcal{D}_{\lambda, \mu}^{m}\left(\alpha_{1}, \beta_{1}\right) F_{c}(f(z))\right]^{\prime}+\gamma z^{2}\left[\mathcal{D}_{\lambda, \mu}^{m}\left(\alpha_{1}, \beta_{1}\right) F_{c}(f(z))\right]^{\prime \prime}}{(1-\gamma) \mathcal{D}_{\lambda, \mu}^{m}\left(\alpha_{1}, \beta_{1}\right) F_{c}(f(z))+\gamma z\left[\mathcal{D}_{\lambda, \mu}^{m}\left(\alpha_{1}, \beta_{1}\right) F_{c}(f(z))\right]^{\prime}} .
$$

A simple computation using (3.1) yields that

$$
z\left(F_{c}(f)(z)\right)^{\prime}+c F_{c}(f)(z)=(c+1) f(z)
$$


and so

$$
\mathcal{D}_{\lambda, \mu}^{m}\left(\alpha_{1}, \beta_{1}\right)\left(z F_{c}(f(z))^{\prime}\right)+c \mathcal{D}_{\lambda, \mu}^{m}\left(\alpha_{1}, \beta_{1}\right) F_{c}(f)(z)=(c+1) \mathcal{D}_{\lambda, \mu}^{m}\left(\alpha_{1}, \beta_{1}\right) f(z) .
$$

By making use of the identity

$$
z\left[\mathcal{D}_{\lambda, \mu}^{m}\left(\alpha_{1}, \beta_{1}\right) F_{c}(f(z))\right]^{\prime}=\mathcal{D}_{\lambda, \mu}^{m}\left(\alpha_{1}, \beta_{1}\right)\left(z F_{c}(f(z))^{\prime}\right)
$$

we get

$$
z\left[\mathcal{D}_{\lambda, \mu}^{m}\left(\alpha_{1}, \beta_{1}\right) F_{c}(f(z))\right]^{\prime}+c \mathcal{D}_{\lambda, \mu}^{m}\left(\alpha_{1}, \beta_{1}\right) F_{c}\left(f(z)=(c+1) \mathcal{D}_{\lambda, \mu}^{m}\left(\alpha_{1}, \beta_{1}\right) f(z)\right.
$$

Differentiating (3.2), we have

$$
\begin{aligned}
& p(z)+c \\
& =(c+1)\left(\frac{(1-\gamma) \mathcal{D}_{\lambda, \mu}^{m}\left(\alpha_{1}, \beta_{1}\right) f(z)+\gamma z\left[\mathcal{D}_{\lambda, \mu}^{m}\left(\alpha_{1}, \beta_{1}\right) f(z)\right]^{\prime}}{(1-\gamma) \mathcal{D}_{\lambda, \mu}^{m}\left(\alpha_{1}, \beta_{1}\right) F_{c}(f(z))+\gamma z\left[\mathcal{D}_{\lambda, \mu}^{m}\left(\alpha_{1}, \beta_{1}\right) F_{c}(f(z))\right]^{\prime}}\right) .
\end{aligned}
$$

Taking logarithmic differentiation of (3.3), we get

$$
p(z)+\frac{z p^{\prime}(z)}{p(z)+c}=\frac{z\left[\mathcal{D}_{\lambda, \mu}^{m}\left(\alpha_{1}, \beta_{1}\right) f(z)\right]^{\prime}+\gamma z^{2}\left[\mathcal{D}_{\lambda, \mu}^{m}\left(\alpha_{1}, \beta_{1}\right) f(z)\right]^{\prime \prime}}{(1-\gamma) \mathcal{D}_{\lambda, \mu}^{m}\left(\alpha_{1}, \beta_{1}\right) f(z)+\gamma z\left[\mathcal{D}_{\lambda, \mu}^{m}\left(\alpha_{1}, \beta_{1}\right) f(z)\right]^{\prime}}
$$

By applying Lemma 2.1 in (3.3), it follows that

$$
p(z) \prec h(z) \quad(z \in U) .
$$

Hence $F_{c}(f) \in A\left(\alpha_{1}, \beta_{1}, \gamma, \lambda, \mu, m, h\right)$.

Special cases of Theroems 3.1 and 3.2 include the results which were given in $[2,11,12,13]$. Interestingly for $q=2, s=1, m=0, \alpha_{1}=\beta_{1}=\alpha_{2}=$ $1, h(z)=\frac{1+z}{1-z}$ and $\gamma=0$ in Theorem 3.1 we obtain $K \subset S^{*}$.

Theorem 3.3. $f \in A\left(\alpha_{1}, \beta_{1}, \gamma, \lambda, \mu, m, h\right)$ if and only if $\gamma z f^{\prime}+(1-\gamma) f \in$ $A\left(\alpha_{1}, \beta_{1}, 0, \lambda, \mu, m, h\right)$.

Proof. Let $f \in A\left(\alpha_{1}, \beta_{1}, \gamma, \lambda, \mu, m, h\right)$ and $g(z)=\gamma z f^{\prime}+(1-\gamma) f$. Using the definition of $\mathcal{D}_{\lambda, \mu}^{m}\left(\alpha_{1}, \beta_{1}\right) f(z)$ and a property of the Hadamard product, we find that $g \in A\left(\alpha_{1}, \beta_{1}, 0, \lambda, \mu, m, h\right)$. Converse is obvious.

Special cases of the Theorem 3.3 includes results which were in $[11,12]$.

Theorem 3.4. If $f \in A\left(\alpha_{1}, \beta_{1}, \gamma, \lambda, \mu, m, h\right)$ then $\gamma f+(1-\gamma) \int_{0}^{z} \frac{f(t)}{t} d t \in$ $A\left(\alpha_{1}, \beta_{1}, 1, \lambda, \mu, m, h\right)$. 
Proof. Let $f \in A\left(\alpha_{1}, \beta_{1}, \gamma, \lambda, \mu, m, h\right)$ then from Theorem 3.3

$$
g(z)=z f^{\prime}+(1-\gamma) f \in A\left(\alpha_{1}, \beta_{1}, 0, \lambda, \mu, m, h\right) .
$$

It can be easily seen that $f \in A\left(\alpha_{1}, \beta_{1}, 1, \lambda, \mu, m, h\right)$ if and only if $z f^{\prime} \in$ $A\left(\alpha_{1}, \beta_{1}, 0, \lambda, \mu, m, h\right)$. Applying this result for $g(z)$, we see that

$$
\gamma f+(1-\gamma) \int_{0}^{z} \frac{f(t)}{t} d t \in A\left(\alpha_{1}, \beta_{1}, 1, \lambda, \mu, m, h\right) .
$$

\section{Theorem 3.5.}

$$
B\left(\alpha_{1}+1, \beta_{1}, \gamma, \lambda, \mu, m, h\right) \subset B\left(\alpha_{1}, \beta_{1}, \gamma, \lambda, \mu, m, h\right) .
$$

Proof. Let $f \in B\left(\alpha_{1}+1, \beta_{1}, \gamma, \lambda, \mu, m, h\right)$ and

$$
p(z)=(1-\gamma) \frac{\mathcal{D}_{\lambda, \mu}^{m}\left(\alpha_{1}, \beta_{1}\right) f(z)}{z}+\gamma\left[\mathcal{D}_{\lambda, \mu}^{m}\left(\alpha_{1}, \beta_{1}\right) f(z)\right]^{\prime} .
$$

Taking $\gamma=1$ in (1.2) we get

$$
z\left[\mathcal{D}_{\lambda, \mu}^{m}\left(\alpha_{1}, \beta_{1}\right) f(z)\right]^{\prime}=\alpha_{1} \mathcal{D}_{\lambda, \mu}^{m}\left(\alpha_{1}+1, \beta_{1}\right) f(z)-\left(\alpha_{1}-1\right) \mathcal{D}_{\lambda, \mu}^{m}\left(\alpha_{1}, \beta_{1}\right) f(z) .
$$

Using (3.5) and the differentiation of (3.5), we get

$$
p(z)+\frac{z p^{\prime}(z)}{\alpha_{1}}=(1-\gamma) \frac{\mathcal{D}_{\lambda, \mu}^{m}\left(\alpha_{1}+1, \beta_{1}\right) f(z)}{z}+\gamma\left[\mathcal{D}_{\lambda, \mu}^{m}\left(\alpha_{1}+1, \beta_{1}\right) f(z)\right]^{\prime} .
$$

By applying Lemma 2.2 in (3.6), we obtain

$$
p(z) \prec q(z) \quad(z \in U) .
$$

Hence the result follows.

Theorem 3.6. If $f \in B\left(\alpha_{1}, \beta_{1}, \gamma, \lambda, \mu, m, h\right)$, then

$$
F_{c}(f) \in B\left(\alpha_{1}, \beta_{1}, \gamma, \lambda, \mu, m, h\right) .
$$

Proof. Assume $f \in B\left(\alpha_{1}, \beta_{1}, \gamma, \lambda, \mu, m, h\right)$ and

$$
p(z)=(1-\gamma) \frac{\mathcal{D}_{\lambda, \mu}^{m}\left(\alpha_{1}, \beta_{1}\right) F_{c}(f(z))}{z}+\gamma\left[\mathcal{D}_{\lambda, \mu}^{m}\left(\alpha_{1}, \beta_{1}\right) F_{c}(f(z))\right]^{\prime} .
$$


Differentiating (3.2) we have

$$
p(z)+\frac{z p^{\prime}(z)}{c+1}=(1-\gamma) \frac{\mathcal{D}_{\lambda, \mu}^{m}\left(\alpha_{1}, \beta_{1}\right) f(z)}{z}+\gamma\left[\mathcal{D}_{\lambda, \mu}^{m}\left(\alpha_{1}, \beta_{1}\right) f(z)\right]^{\prime} .
$$

By applying Lemma 2.2 in (3.7) we get

$$
p(z) \prec h(z)
$$

and hence

$$
F_{c}(f) \in B\left(\alpha_{1}, \beta_{1}, \gamma, \lambda, \mu, m, h\right) .
$$

Theorem 3.7. $C\left(\alpha_{1}+1, \beta_{1}, \gamma, \lambda, \mu, m, h\right) \subset C\left(\alpha_{1}, \beta_{1}, \gamma, \lambda, \mu, m, h\right)$.

Proof. Let $f \in C\left(\alpha_{1}+1, \beta_{1}, \gamma, \lambda, \mu, m, h\right)$ and

$$
p(z)=\left[\mathcal{D}_{\lambda, \mu}^{m}\left(\alpha_{1}, \beta_{1}\right) f(z)\right]^{\prime}+\gamma z\left[\mathcal{D}_{\lambda, \mu}^{m}\left(\alpha_{1}, \beta_{1}\right) f(z)\right]^{\prime \prime} .
$$

Differentiating (3.5), we have

$$
p(z)+\frac{z p^{\prime}(z)}{\alpha_{1}}=\left[\mathcal{D}_{\lambda, \mu}^{m}\left(\alpha_{1}+1, \beta_{1}\right) f(z)\right]^{\prime}+\gamma z\left[\mathcal{D}_{\lambda, \mu}^{m}\left(\alpha_{1}+1, \beta_{1}\right) f(z)\right]^{\prime \prime} .
$$

Applying Lemma 2.2 in (3.8), we get

$$
p(z) \prec h(z) \quad(z \in U)
$$

and the result now follows.

Theorem 3.8. If $f \in C\left(\alpha_{1}, \beta_{1}, \gamma, \lambda, \mu, m, h\right)$, then

$$
F_{c}(f) \in C\left(\alpha_{1}, \beta_{1}, \gamma, \lambda, \mu, m, h\right) .
$$

Proof. Let $f \in C\left(\alpha_{1}, \beta_{1}, \gamma, \lambda, \mu, m, h\right)$ and

$$
p(z)=\left[\mathcal{D}_{\lambda, \mu}^{m}\left(\alpha_{1}, \beta_{1}\right) F_{c}(f(z))\right]^{\prime}+\gamma z\left[\mathcal{D}_{\lambda, \mu}^{m}\left(\alpha_{1}, \beta_{1}\right) F_{c}(f(z))\right]^{\prime \prime} .
$$

Differentiating (3.2) we get

$$
p(z)+\frac{z p^{\prime}(z)}{c+1}=\left[\mathcal{D}_{\lambda, \mu}^{m}\left(\alpha_{1}, \beta_{1}\right) f(z)\right]^{\prime}+\gamma z\left[\mathcal{D}_{\lambda, \mu}^{m}\left(\alpha_{1}, \beta_{1}\right) f(z)\right]^{\prime \prime} .
$$

A simple application of Lemma 2.2 will give the desired result. 
Theorem 3.9. $f \in C\left(\alpha_{1}, \beta_{1}, \gamma, \lambda, \mu, m, h\right)$ if and only if

$$
z f^{\prime} \in B\left(\alpha_{1}, \beta_{1}, \gamma, \lambda, \mu, m, h\right) .
$$

Proof. Using the equality

$$
z\left[\mathcal{D}_{\lambda, \mu}^{m}\left(\alpha_{1}, \beta_{1}\right) f(z)\right]^{\prime}=\mathcal{D}_{\lambda, \mu}^{m}\left(\alpha_{1}, \beta_{1}\right)\left(z f^{\prime}(z)\right) .
$$

We see that

$$
\begin{aligned}
(1-\gamma) \frac{\mathcal{D}_{\lambda, \mu}^{m}\left(\alpha_{1}, \beta_{1}\right) z f^{\prime}(z)}{z}+\gamma & {\left[\mathcal{D}_{\lambda, \mu}^{m}\left(\alpha_{1}, \beta_{1}\right) z f^{\prime}(z)\right]^{\prime} } \\
& =\left[\mathcal{D}_{\lambda, \mu}^{m}\left(\alpha_{1}, \beta_{1}\right) f(z)\right]^{\prime}+\gamma z\left[\mathcal{D}_{\lambda, \mu}^{m}\left(\alpha_{1}, \beta_{1}\right) f(z)\right]^{\prime \prime}
\end{aligned}
$$

which implies the required result.

Theorem 3.10. $C\left(\alpha_{1}, \beta_{1}, \gamma, \lambda, \mu, m, h\right) \subset B\left(\alpha_{1}, \beta_{1}, \gamma, \lambda, \mu, m, h\right)$.

Proof. Let $f \in C\left(\alpha_{1}, \beta_{1}, \gamma, \lambda, \mu, m, h\right)$ and

$$
p(z)=(1-\gamma) \frac{\mathcal{D}_{\lambda, \mu}^{m}\left(\alpha_{1}, \beta_{1}\right) f(z)}{z}+\gamma\left[\mathcal{D}_{\lambda, \mu}^{m}\left(\alpha_{1}, \beta_{1}\right) f(z)\right]^{\prime} .
$$

Hence

$$
p(z)+z p^{\prime}(z)=\left[\mathcal{D}_{\lambda, \mu}^{m}\left(\alpha_{1}, \beta_{1}\right) f(z)\right]^{\prime}+\gamma z\left[\mathcal{D}_{\lambda, \mu}^{m}\left(\alpha_{1}, \beta_{1}\right) f(z)\right]^{\prime \prime}
$$

and the result follows as an application of Lemma 2.2.

Theorem 3.11. For $\gamma>\delta \geq 0$,

$$
B\left(\alpha_{1}, \beta_{1}, \gamma, \lambda, \mu, m, h\right) \subset B\left(\alpha_{1}, \beta_{1}, \delta, \lambda, \mu, m, h\right) .
$$

Proof. Let $f \in B\left(\alpha_{1}, \beta_{1}, \gamma, \lambda, \mu, m, h\right)$ and $p(z)=\frac{\mathcal{D}_{\lambda, \mu}^{m}\left(\alpha_{1}, \beta_{1}\right) f(z)}{z}$. When $\delta=0$, we have

$$
p(z)+z p^{\prime}(z)=(1-\gamma) \frac{\mathcal{D}_{\lambda, \mu}^{m}\left(\alpha_{1}, \beta_{1}\right) f(z)}{z}+\gamma\left[\mathcal{D}_{\lambda, \mu}^{m}\left(\alpha_{1}, \beta_{1}\right) f(z)\right]^{\prime}
$$

Hence the result follows as an application of Lemma 2.2 in (3.10), when $\delta=0$. Suppose $\delta \neq 0$. Since $f \in B\left(\alpha_{1}, \beta_{1}, \gamma, \lambda, \mu, m, h\right)$, we have

$$
(1-\gamma) \frac{\mathcal{D}_{\lambda, \mu}^{m}\left(\alpha_{1}, \beta_{1}\right) f(z)}{z}+\gamma\left[\mathcal{D}_{\lambda, \mu}^{m}\left(\alpha_{1}, \beta_{1}\right) f(z)\right]^{\prime} \in h(U) \quad(z \in U) .
$$


But $\frac{\mathcal{D}_{\lambda, \mu}^{m}\left(\alpha_{1}, \beta_{1}\right) f(z)}{z} \in h(U)$ and $h(U)$ is convex. Also

$$
\begin{aligned}
(1-\delta) \frac{\mathcal{D}_{\lambda, \mu}^{m}\left(\alpha_{1}, \beta_{1}\right) f(z)}{z}+ & \delta\left[\mathcal{D}_{\lambda, \mu}^{m}\left(\alpha_{1}, \beta_{1}\right) f(z)\right]^{\prime}=\left(1-\frac{\delta}{\gamma}\right) \frac{\mathcal{D}_{\lambda, \mu}^{m}\left(\alpha_{1}, \beta_{1}\right) f(z)}{z} \\
+ & \frac{\delta}{\gamma}\left[(1-\gamma) \frac{\mathcal{D}_{\lambda, \mu}^{m}\left(\alpha_{1}, \beta_{1}\right) f(z)}{z} \gamma\left[\mathcal{D}_{\lambda, \mu}^{m}\left(\alpha_{1}, \beta_{1}\right) f(z)\right]^{\prime}\right]
\end{aligned}
$$

Therefore we have

$$
(1-\delta) \frac{\mathcal{D}_{\lambda, \mu}^{m}\left(\alpha_{1}, \beta_{1}\right) f(z)}{z}+\delta\left[\mathcal{D}_{\lambda, \mu}^{m}\left(\alpha_{1}, \beta_{1}\right) f(z)\right]^{\prime} \in h(U) .
$$

Hence the result follows.

Theorem 3.12. For $\gamma>\delta \geq 0$,

$$
C\left(\alpha_{1}, \beta_{1}, \gamma, \lambda, \mu, m, h\right) \subset C\left(\alpha_{1}, \beta_{1}, \delta, \lambda, \mu, m, h\right)
$$

Proof. Let $f(z) \in C\left(\alpha_{1}, \beta_{1}, \gamma, \lambda, \mu, m, h\right)$ and $p(z)=\left[\mathcal{D}_{\lambda, \mu}^{m}\left(\alpha_{1}, \beta_{1}\right) f(z)\right]^{\prime}$. When $\delta=0$, we have

$$
p(z)+\gamma z p^{\prime}(z)=\left[\mathcal{D}_{\lambda, \mu}^{m}\left(\alpha_{1}, \beta_{1}\right) f(z)\right]^{\prime}+\gamma z\left[\mathcal{D}_{\lambda, \mu}^{m}\left(\alpha_{1}, \beta_{1}\right) f(z)\right]^{\prime \prime} .
$$

Hence the result follows as an application of Lemma 2.2, when $\delta=0$.

Suppose $\delta \neq 0$. Then

$$
\left[\mathcal{D}_{\lambda, \mu}^{m}\left(\alpha_{1}, \beta_{1}\right) f(z)\right]^{\prime}+\gamma z\left[\mathcal{D}_{\lambda, \mu}^{m}\left(\alpha_{1}, \beta_{1}\right) f(z)\right]^{\prime \prime} \in h(U) \quad(z \in U) .
$$

Note that

$$
\begin{aligned}
& {\left[\mathcal{D}_{\lambda, \mu}^{m}\left(\alpha_{1}, \beta_{1}\right) f(z)\right]^{\prime}+\delta z\left[\mathcal{D}_{\lambda, \mu}^{m}\left(\alpha_{1}, \beta_{1}\right) f(z)\right]^{\prime \prime} } \\
= & \left(1-\frac{\delta}{\gamma}\right)\left[\mathcal{D}_{\lambda, \mu}^{m}\left(\alpha_{1}, \beta_{1}\right) f(z)\right]^{\prime}+\frac{\delta}{\gamma}\left[\mathcal{D}_{\lambda, \mu}^{m}\left(\alpha_{1}, \beta_{1}\right) f(z)\right]^{\prime}+\gamma z\left[\mathcal{D}_{\lambda, \mu}^{m}\left(\alpha_{1}, \beta_{1}\right) f(z)\right]^{\prime \prime} .
\end{aligned}
$$

As $h(U)$ is convex and $\frac{\delta}{\gamma}<1$ we have

$$
\left[\mathcal{D}_{\lambda, \mu}^{m}\left(\alpha_{1}, \beta_{1}\right) f(z)\right]^{\prime}+\delta z\left[\mathcal{D}_{\lambda, \mu}^{m}\left(\alpha_{1}, \beta_{1}\right) f(z)\right]^{\prime \prime} \in h(U) \quad(z \in U)
$$

and hence the result follows. 


\section{References}

[1] F. M. Al-Oboudi, On univalent functions defined by a generalized Sălăgean operator, Int. J. Math. Math. Sci., 27 (2004), 1429-1436.

[2] S D Bernardi, Convex and starlike univalent functions, Trans. Amer. Math. Soc., 135 (1969), 429-446.

[3] B C Carlson, D B Shaffer, Starlike and prestarlike hypergeometric fucntions, SIAM. J. Math. Anal., 15, No. 4 (1984), 737-745.

[4] Dorina Răducanu, Halit Orhan, Subclasses of analytic functions defined by a generalized differential operator, Intern. J. Math. Anal., 4, No. 1 (2010), 1-15.

[5] P L Duren, Univalent functions - A series of comprehensive studies in Mathematics, Springer-Verlag, Japan(1983).

[6] J Dziok and H M Srivatsava, Classes of analytic functions associated with the generalized hypergeometric function, Appl. Math. Comput., 103, No. 1 (1999), 1- 13.

[7] Ju. E Hohlov, Operators and operations on the class of univalent functions, Izv. Vyssh. Uchebn. Zaved. Math., 10, No. 197 (1978), 83-89.

[8] R J Libera, Some classes of regular univalent functons, Proc. Amer. Math. Soc., 16 (1965), 755-758.

[9] N Marikkannan, A subclass of analytic functions and a generalised differential operator, communicated.

[10] S S Miller and P T Mocanu, Differential subordinations: Theory and applications, Marcel Dekker, New york, (2000).

[11] Öznur Özkan and Osman Altintas, Applications of Differential subordination, Appl. Math. Compu.(19),(2006),728-734.

[12] K S Padmanabhan and R Manjini, Certain applications of differential subordination, Publ. Insti. Math (N. S), Tome 39, No. 53), (1986), 107-118.

[13] K S Padmanabhan and R Parvatham, Some applications of differential subordination, Bull. Austral. Math. Soc., 32 (1985), 321-330. 
[14] G. Ş. Sălăgean, Subclasses of univalent functions, Complex analysis-fifth Romanian-Finnish seminar, Part 1 (Bucharest, 1981), 362-372, Lecture Notes in Math., 1013 Springer, Berlin.

[15] C Selvaraj, K R Karthikeyan, Subclasses of analytic functions involving a certain family of linear operators, Int. J. Contemp. Math. Sci., 3, No. 13 (2008), 615-627. 